Research Article

\title{
Prevalence and Genetic Relationship of Predominant Escherichia coli Serotypes Isolated from Poultry, Wild Animals, and Environment in the Mekong Delta, Vietnam
}

\author{
Lam Thanh Nguyen $\mathbb{D}^{1},{ }^{1}$ Nguyen Khanh Thuan $\mathbb{D}^{1},{ }^{1}$ Nguyen Thu Tam, ${ }^{1}$ \\ Chau Thi Huyen Trang, ${ }^{1}$ Nguyen Phuc Khanh, ${ }^{1}$ Tran Ngoc Bich, ${ }^{1}$ Takahide Taniguchi, \\ Hideki Hayashidani ${ }^{2}{ }^{2}$ and Ly Thi Lien Khai ${ }^{1}{ }^{1}$ \\ ${ }^{1}$ Department of Veterinary Medicine, College of Agriculture, Can Tho University, Campus II, 3/2 Street, Ninh Kieu District, \\ Can Tho, Vietnam \\ ${ }^{2}$ Division of Animal Life Science, Institute of Agriculture, Tokyo University of Agriculture and Technology, \\ 3-5-8 Saiwai-Cho, Fuchu-Shi, Tokyo 183-8509, Japan
}

Correspondence should be addressed to Lam Thanh Nguyen; ntlam@ctu.edu.vn and Ly Thi Lien Khai; ltlkhai@ctu.edu.vn Received 13 September 2021; Accepted 13 October 2021; Published 11 November 2021

Academic Editor: Juan G. Chediack

Copyright (c) 2021 Lam Thanh Nguyen et al. This is an open access article distributed under the Creative Commons Attribution License, which permits unrestricted use, distribution, and reproduction in any medium, provided the original work is properly cited.

\begin{abstract}
Avian pathogenic Escherichia coli (APEC) is the main causative agent of avian colibacillosis, which is an important systemic disease of profound economic and clinical consequences for the poultry industry worldwide. In this study, 975 E. coli strains were isolated from 2,169 samples collected from cloacal swabs of chickens, in-farm wild animals (ants, geckos, flies, and rats), and environment. The highest proportion of E. coli isolation was obtained from chicken cloacal swabs with $71.05 \%$ (95\% confidence interval (CI) $66.69-75.05 \%$ ) followed by the proportions of $38.15 \%$ (95\% CI $35.41-40.97 \%)$ and $38.11 \%$ (95\% CI 34.15-42.24\%) from wild animals or environment, respectively. Distribution of $\mathrm{O}$-antigen serotypes of the E. coli isolates, including $\mathrm{O} 1, \mathrm{O} 2, \mathrm{O} 18$, and O78, was determined by PCR. The most predominant serotype was O18 (10.56\%) followed by O2 (9.44\%), O1 (7.79\%), and O78 (6.56\%). Of note, serotype O18 was more likely distributed in the examined wild animals, especially in geckos. Polymorphic DNA fingerprints, generated by ERIC-PCR, of representative E. coli strains of each serotype revealed genetic heterogeneity of the examined E. coli, and O18 was more divergent with 63 clusters formed from 66 isolates. Furthermore, several E. coli strains from different sample sources shared high DNA fingerprint relatedness, suggesting that there exists complex transmission of $E$. coli from chickens to wild animals and environment and vice versa in poultry husbandry settings. Although pathotypes of the examined E. coli were not determined in this study, our results provided important findings of epidemiological and genetic characteristics of E. coli in the Mekong Delta and highlighted the prerequisite of stricter biocontainment to reduce the prevalence and consequences of APEC in poultry production.
\end{abstract}

\section{Introduction}

Escherichia coli (E. coli) is a ubiquitous organism in the gastrointestinal microbiota of human and animals. A majority of $E$. coli are nonpathogenic and play an important role in host metabolism, immunology, and nutrition [1]. However, several E. coli strains can acquire specific virulence factors and become pathogenic E. coli that are capable of causing a wide range of diseases in human and animals [2]. Concerning animal health, avian pathogenic E. coli (APEC) is the primary cause of avian colibacillosis; the disease is characterized by multiple systemic syndromes such as colisepticemia, airsacculitis, perihepatitis, pericarditis, swollenhead syndrome, and fatal hemorrhagic septicemia, resulting in high morbidity, mortality, and carcass condemnation [2]. Thus, APEC is responsible for severe economic and clinical 
consequences to poultry production worldwide [3]. Besides the serious impacts on poultry health, many recent studies have indicated that a subset of APEC might cause zoonotic infections, posing a real threat to public health [4-7].

E. coli could be serotyped by differentiating its somatic $\mathrm{O}$ (a component of the surface lipopolysaccharide) and $\mathrm{H}$ (flagellar) antigens; thus, variability of the O-antigen provides the basis for many serotyping schemes and that becomes the current standard classification of taxonomy and epidemiology of E. coli $[8,9]$. At present, 181 O-serotypes of E. coli, numbered from $\mathrm{O} 1$ to $\mathrm{O} 181$, have been recognized [8-10], in which several specific O-serotypes of APEC have been frequently confirmed to be closely associated with serious diseases in human and animals. Notably, certain APEC belonging to the serotypes $\mathrm{O} 1, \mathrm{O} 2, \mathrm{O} 18$, and $\mathrm{O} 78$ have been considered the most common pathogenic strains and that is accounted for more than $80 \%$ of the APEC isolates [11-13].

Several studies have been carried to investigate the microbiological characterization of APEC, including the distribution of serotypes, attributed virulence, antimicrobial resistance, and phylogeny in several major poultry producing countries such as Spain [14], China [15], and South Korea [16]. However, little knowledge has been elucidated about epidemiological and genetic characteristics of APEC in poultry production in Vietnam where there exists a rapid transition to large and/or industrial-scale poultry enterprises [17]. Therefore, with this background, this study was conducted (i) to determine the prevalence and distribution of common serotypes of APEC in poultry, in-farm wild animals, and environment and (ii) to identify the genetic relationship of E. coli strains among these sample sources.

\section{Materials and Methods}

2.1. Ethical Approval. All experimental protocols were approved by the Institutional Animal Care and Use Committee of Can Tho University, Vietnam. Capture and dissection of wild animals were ethically performed following the guideline in accordance with the Regulation on Animal Experimentation of Can Tho University.

2.2. Sampling Site, Period, and Sample Collection. The sampling procedure was performed in backyards/small poultry farms in Vinh Long Province covering an area of about $1,526 \mathrm{~km}^{2}$ with approximately 9.6 million chickens [18], and this province is considered as a central division for poultry production in the Mekong Delta, Vietnam (Supplementary Figure 1). From 2018 to 2020, a total of 2,169 samples including cloacal swabs from healthy chickens, infarm wild animals including ants, geckos (house gecko, Hemidactylus frenatus, flat-tailed house gecko, Hemidactylus platyurus, and four-clawed gecko, Gehyra mutilata), flies (housefly, Musca domestica, and blowfly, Calliphoridae), and rats, and environmental samples were collected in this study. Details of sample types and distribution are described in Supplementary Table 1. All swab samples (cloaca feces; barn floors) were put into a Carry-Blair transport medium
(Merck, Germany). Wild animals were trapped and put separately in sterile bags with ventilation holes; feed (250 g) and drinking water $(1,000 \mathrm{~mL})$ were collected directly in the flocks. All samples were cooled in an icebox and immediately transported to the laboratory within 24 hours for processing and identification.

In the laboratory, geckos were euthanized by freezing at $-20^{\circ} \mathrm{C}$ in five minutes, while rats were euthanized by using chloroform (Merck, Germany). Both were dissected at room temperature to collect cecal content individually and aseptically. Ants and flies were also inactivated by freezing at $-20^{\circ} \mathrm{C}$ in five minutes, and all bodies were suspended in the enrichment broth of buffered peptone water medium (BPW, Merck, Germany).

2.3. E. coli Isolation and Identification. Isolation and identification of $E$. coli were performed in accordance with Vietnam National Standard of TCVN 7924-2:2008 [19], equivalent to the standard colony morphology and biochemical identification methods as previously described [20]. Briefly, collected samples were put into the enrichment broth and buffered peptone water medium (BPW, Merck, Germany) and incubated for 24 hours at $37^{\circ} \mathrm{C}$. Then, the enriched suspension was inoculated onto MacConkey agar (MC, Merck, Germany) and incubated at $37^{\circ} \mathrm{C}$ overnight. From each positive sample, ten suspicious $E$. coli colonies were selected for subculture on nutrient agar (NA, Merck, Germany). After incubation for 24 hours at $37^{\circ} \mathrm{C}$, those isolates were individually examined for biochemical tests as previously described [21].

2.4. DNA Extraction. Total genomic DNA of a single E. coli strain was extracted by boiling method. A loopful of a colony on the nutrient agar (NA, Merck, Germany) was dissolved with $1 \mathrm{~mL}$ of sterile deionized distilled water and vortexed for $10 \mathrm{~s}$. The suspension was placed on a thermal block at $95^{\circ} \mathrm{C}$ for $10 \mathrm{~min}$ and then was centrifuged at $10,000 \mathrm{rpm}$ for $5 \mathrm{~min}$. The supernatant from this suspension was used as the template for PCR [22].

2.5. Serotyping Using Molecular PCR Detection. All the single E. coli isolates were serotyped using conventional single PCR with four primer pairs that were previously developed to detect and differentiate each of serotypes O1, O2, O18, and O78 [23]. Each $20 \mu \mathrm{l}$ PCR reaction mixture comprises $1 \mu \mathrm{l}$ of genomic DNA template and $18 \mu \mathrm{l}$ of the $2 \mathrm{x}$ colorless Go-Taq master mix that included $\mathrm{MgCl}_{2}, 10 \mathrm{x}$ PCR buffer, dNTPs, 10 units of Taq DNA polymerase (Cat \#M7132, Promega, Madison, WI, USA), and $0.5 \mu \mathrm{l}$ of each $10 \mu \mathrm{M}$ forward and reverse primer. PCR amplification was performed under the following reaction conditions: initial denaturation at $94^{\circ} \mathrm{C}$ for $5 \mathrm{~min}$, followed by 30 cycles of $95^{\circ} \mathrm{C}$ for $35 \mathrm{~s}, 57^{\circ} \mathrm{C}$ for $30 \mathrm{~s}$, $72^{\circ} \mathrm{C}$ for $1 \mathrm{~min}$, a final extension at $72^{\circ} \mathrm{C}$ for $10 \mathrm{~min}$, and then holding at $4^{\circ} \mathrm{C}$. The resulting PCR products were visualized on $1.5 \%$ tris-acetate-EDTA agarose gels stained with ethidium bromide alongside a $100 \mathrm{bp}$ ladder (Bioline, UK) and photographed under UV transillumination. 
2.6. ERIC-PCR and Fingerprints Analyses. Representative $E$. coli isolates of a single colony per sample were fingerprinted using the enterobacterial repetitive intergenic consensus- (Eric-) PCR. A set of primers ERIC-1 (5'-ATG TAA GCT CCT GGG GAT TCA C-3') and ERIC-2 (5' -AAG TAA GTG ACT GGG GTG AGC G-3') was used to amplify the regions in the bacterial genome positioned between the Eric sequences [24]. The component of the ERIC-PCR reaction mixture was prepared as mentioned above. The ERIC-PCR was performed in a thermocycler under the following condition: initial denaturation at $94^{\circ} \mathrm{C}$ for $5 \mathrm{~min}$ followed by 35 cycles consisting of denaturation at $94^{\circ} \mathrm{C}$ for $1 \mathrm{~min}$, annealing at $50^{\circ} \mathrm{C}$ for $1 \mathrm{~min}$, extension at $65^{\circ} \mathrm{C}$ for $8 \mathrm{~min}$, a final extension step at $65^{\circ} \mathrm{C}$ for $8 \mathrm{~min}$, and final storage at $4^{\circ} \mathrm{C}$. The products of ERIC-PCR were electrophoresed on a $1.5 \%$ agarose gel, then stained with ethidium bromide, and visualized by UV transillumination. The image was captured using a gel documentation system for further analysis of DNA fingerprinting.

Polymorphic DNA fingerprint was analyzed using Bionumerics software version 7.0 (Applied Maths, SintMartens-Latem, Belgium). Four dendrograms showing clustering of the APEC strains of each serotype were generated based on the averaged similarity of the matrix with the use of the algorithm of the Unweighted Pair Group Method with Arithmetic Mean (UPGMA) analysis and Dice similarity coefficient [24]. A cutoff value of $\geq 85 \%$ similarity coefficient was applied to assign the clusters [25].

2.7. Data Execution and Statistical Analysis. Data collection was undertaken using Microsoft Excel. The prevalence proportion (expressed as a percentage) of E. coli isolation was determined, and its confidence intervals (CI) were calculated as $95 \%$ binomial proportions representing Wilson score intervals. Statistical analyses, graphing, and visualization were performed through computing platform $\mathrm{R}$ version 4.0.1 [26], contributed packages ggplot2 [27], binom [28], and epiR [29].

\section{Results}

3.1. Prevalence of E. coli. A total of 975 E. coli strains were isolated from 2,169 samples collected from cloacal swabs of chickens, in-farm wild animals (ants, geckos, flies, and rats), and environment. The proportions and the CIs of E. coli isolates per sample are presented in Figure 1. E. coli was isolated from 319 of 449 (71.05\% (95\% CI 66.69-75.05\%)) cloacal swab samples of chickens, which was significantly higher than the number of $E$. coli either isolated from wild animals 446 of 1,169 (38.15\% (95\% CI 35.41-40.97\%)) and environment 210 of 551 (38.11\% (95\% CI 34.15-42.24\%)), respectively (Figure 1(a)). Within the studied wild animals, the number of $E$. coli isolated from rats was 35 of $52(67.30 \%$ (95\% CI 53.76-78.48\%)) that was the highest proportion in comparison with geckos 278 of $620(44.84 \%$ (95\% CI $40.97-48.77 \%))$, flies 108 of $297 \quad(36.36 \% \quad(95 \% \quad$ CI $31.10-41.98 \%)$ ), and ants 25 of $200(12.50 \%$ (95\% CI 8.61-17.80\%)), respectively (Figure 1(b)).
3.2. Distribution of Serotypes O1, O2, O18, and O78 in E. coli Isolates. All 975 E. coli strains were serotyped with $\mathrm{O} 1, \mathrm{O} 2$, O18, and O78 using conventional PCR (Supplementary Figure 2). The distribution of the examined O-serotypes is shown in Figure 2. Proportions of E. coli belonging to the serotypes $\mathrm{O} 1, \mathrm{O} 2$, O18, and $\mathrm{O} 78$ were $7.79 \%$, 9.44\%, 10.56\%, and $6.56 \%$, respectively. A large remaining proportion (65.64\%) of E. coli was unknown serotypes (Figure 2(a)). In chickens, the distribution of $\mathrm{O} 1, \mathrm{O} 2, \mathrm{O} 18$, and $\mathrm{O} 78 \mathrm{E}$. coli was almost equivalent with the proportions of $11.29 \%(95 \%$ CI 8.26-15.22\%), 9.72\% (95\% CI 6.93-13.46\%), 7.84\% (95\% CI $5.36-11.31 \%$ ), and $8.46 \%$ (95\% CI $5.88-12.03 \%)$, respectively. Similarly, the presence of these four serotypes was comparable in the environment samples with proportions ranging from 2.86\% (O1) to 6.19\% (O2) (Figure 2(b)). Remarkably, $\mathrm{O} 18$ E. coli was accounted for a relatively large proportion of $15.47 \%$ (95\% CI 12.41-19.12\%) of E. coli isolated from wild animals, and the majority of the $\mathrm{O} 18$ E. coli were isolated from in-farm geckos (46.39\%), $45 \mathrm{O} 18$ E. coli isolates from geckos out of total $97 \mathrm{O} 18 \mathrm{E}$. coli isolates in all collected samples (data not shown).

3.3. Genomic DNA Fingerprinting and Genetic Relationship of O1, O2, O18, and 078 E. coli. Representative E. coli isolates of serotypes O1 (42 isolates), O2 (39 isolates), O18 (66 isolates), and O78 (39 isolates) were selected based on sample types and space-time sources for ERIC-PCR to identify genetic divergence and relationship among the $E$. coli isolates. Four dendrograms generated from genomic fingerprinting of $E$. coli isolates belonging to the examined serotypes were, respectively, described in Figures 3(a)-3(d). These results showed that DNA fingerprinting patterns of E. coli were heterogeneous, which was characterized by allocation of multiple genetic subclusters per serotype: $\mathrm{O} 1$ (38 clusters out of 42 isolates), O2 (35 clusters out of 39 isolates), $\mathrm{O} 18$ (64 clusters out of 66), and O78 (35 clusters out of 39 isolates) and all these subclusters were grouped into two (O78) to three $(\mathrm{O} 1, \mathrm{O} 2$, and $\mathrm{O} 18)$ major clusters. Importantly, within the same serotype, several E. coli strains isolated from different sample sources shared high genetic similarity $(\geq 85 \%)$ and grouped together into distinct subclusters, for example, O1 serotypes (P4-O1-C/P5-O1-G, P18-O1-C/P19-O1-E, and P37-O1-C/P38-O1-E); O2 serotype (P9-O2-C/P10-O2-E, P12-O2-C/P13-O2-E, and P28-O2-G/P29-O2-C), O18 serotype (P23-O18-R/ $\mathrm{P} 24-\mathrm{O} 18-\mathrm{G})$, and O78 serotype (P17-O78-E/P18-O78-C and P35-O78-C/P36-O78-E).

\section{Discussion}

Avian pathogenic E. coli is the major causative agent of avian colibacillosis that is considered one of the most important bacterial diseases in the global poultry industry due to its substantial economic impacts $[2,30]$. In addition to causing serious consequences on poultry health and production, several APEC strains have been determined as potential zoonotic pathogens [4-7]. Therefore, comprehensive knowledge of biological and epidemiological characteristics 


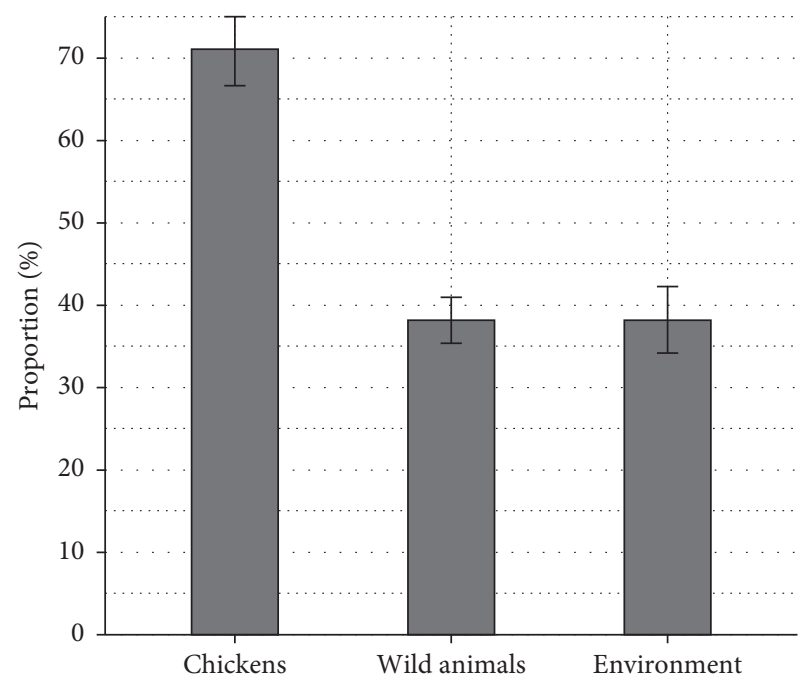

(a)

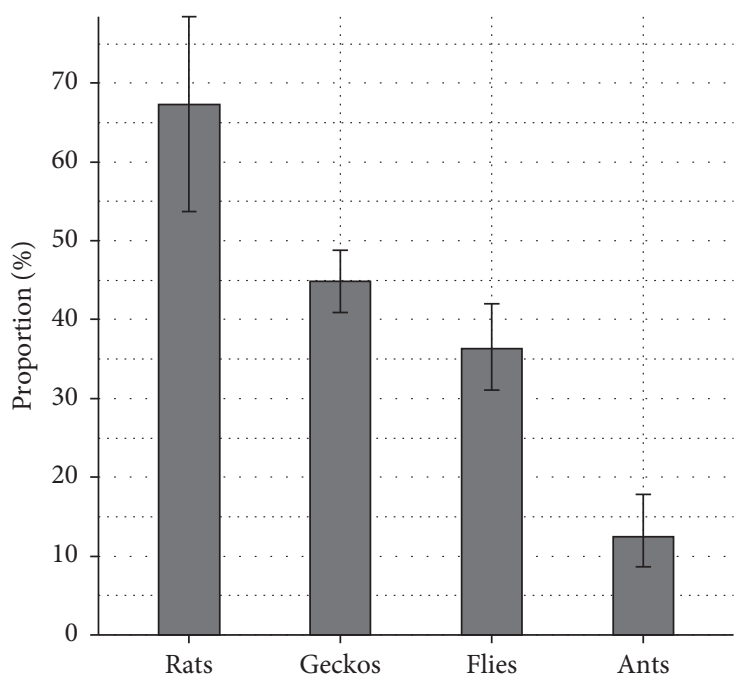

(b)

FIGURE 1: Bar graphs showing the proportion of E. coli isolated from cloacal swabs of chickens, wild animals, and environment (a) and proportion of E. coli isolated from ants, geckos, flies, and rats (b). The error bar indicates $95 \%$ confidence interval.

of APEC is critical to reducing the incidence of avian colibacillosis in poultry production and threats to human health. In this study, we first carried out isolation and identification of $E$. coli collected from chickens, wild animals, and environment. Then, the obtained $E$. coli isolates were serotyped using PCR targeting $\mathrm{O}$-antigen genes of the notable $\mathrm{O} 1, \mathrm{O} 2, \mathrm{O} 18$, and $\mathrm{O} 78$ serotypes, and finally, the genetic relationship of these $E$. coli was examined using DNA fingerprints generated from ERIC-PCR.

A total of $975 \mathrm{E}$. coli strains were isolated from 2,169 samples (44.95\% isolation rate) from chickens, wild animals, and environment. The highest isolation rate was from chicken samples (71.05\% (95\% CI 66.69-75.05\%)) as compared with the wild animals and environment samples (Figure 1). Among wild animals, relatively high isolation rates were also obtained from rats $(67.31 \%(95 \% \mathrm{CI}$ $53.76-78.48 \%))$ and geckos $(44.84 \% \quad(95 \% \quad \mathrm{CI}$ $40.97-48.77 \%)$ ). This result is consistent with the common biological plausibility that $E$. coli coexist in the gastrointestinal tract as harmless commensal symbionts in warmblooded animals [1]. Nevertheless, the high prevalence of E. coli recovered from chickens, one of the major reservoirs of APEC, and in-farm wild animals might be an issue for poultry husbandry since a subset of otherwise commensal E. coli strains derived from the animals could be pathogenic E. coli and potentially implicate localized or systemic infections when birds suffer from immune suppression, stress, or injury [2].

A few studies have been done to elucidate microbiological characteristics of $E$. coli existing in poultry production in Vietnam. Most studies focused on investigating virulence attributes and/or antibiotics resistance of $E$. coli strains obtained from either poultry host species and environment [31, 32]; however, information about the distribution of serotypes of such E. coli remains elusive. In this study, all 975 E. coli strains were subjected to serotyping using conventional PCR. As literature review, APEC serotypes $\mathrm{O} 1, \mathrm{O} 2, \mathrm{O} 18$, and $\mathrm{O} 78$ were targets for the present serotyping since it has been widely reported that $E$. coli strains in these serotypes are the most common APEC pathotypes causing colibacillosis in animals and human $[7,33]$. A total of 335 E. coli out of $975(34.35 \%)$ E. coli strains were defined as belonging to the serotypes $\mathrm{O} 1, \mathrm{O} 2, \mathrm{O} 18$, and O78 (Figure 2(a)). These serotypes were distributed at almost equivalent proportions among chickens, wild animals, and environment (Figure 2(b)). This is in line with previous studies showing that the prevalence of $E$. coli ranges from $9.52 \%$ to $36.73 \%$ in all age groups of chickens [34]. Remarkably, serotype $\mathrm{O} 18$ was more likely predominant with the highest proportion at $10.56 \%$ followed by O2 (9.44\%), O1 (7.79\%), and O78 (5.56\%). The higher proportion of the $\mathrm{O} 18$ E. coli gives greater consideration since certain $\mathrm{O} 18$ APEC strains have been reported to have zoonotic importance of causing urinary tract infections and meningitis in humans [35]. On the other hand, 45 isolates, out of the total 97 (46.39\%) O18 E. coli isolates recovered from all samples, were derived from geckos. Several previous studies also raise concerns about geckos' role in disease transmission where the species can be either natural reservoirs and transmitters for pathogenic bacteria such as APEC and Salmonella $[16,36-38]$. It is also noteworthy to mention that more than half $(65.64 \%)$ of the E. coli isolates were not assigned their serotypes. There might be important $E$. coli strains linked to other APEC pathotypes and should be included for further serotyping targets in order to provide explicit information about the population characteristics of APEC.

ERIC-PCR was employed to determine molecular fingerprints of examined E. coli since this technique is reproducible and cost-effective and has been widely used to evaluate the genetic relatedness of bacteria from different origins [39]. Dendrograms generated from ERIC-PCR fingerprints of $42 \mathrm{O} 1$ isolates, $39 \mathrm{O} 2$ isolates, $66 \mathrm{O} 18$ isolates, 


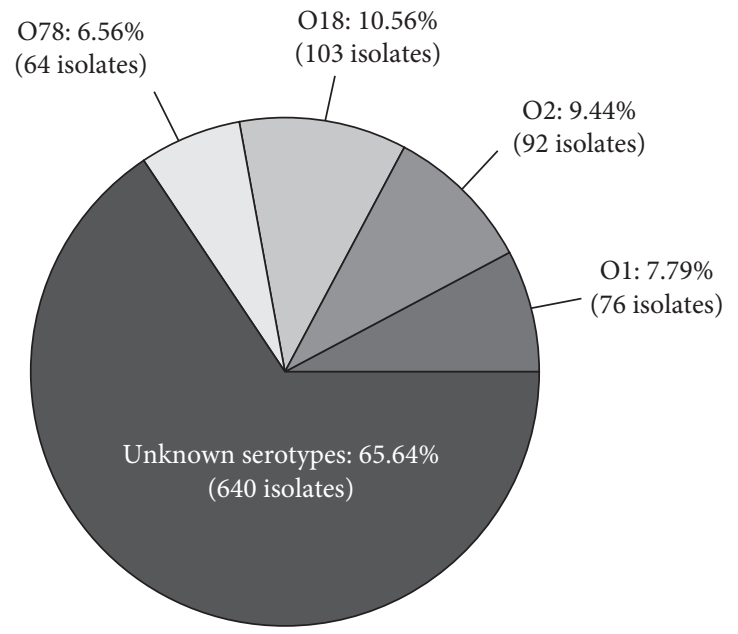

(a)

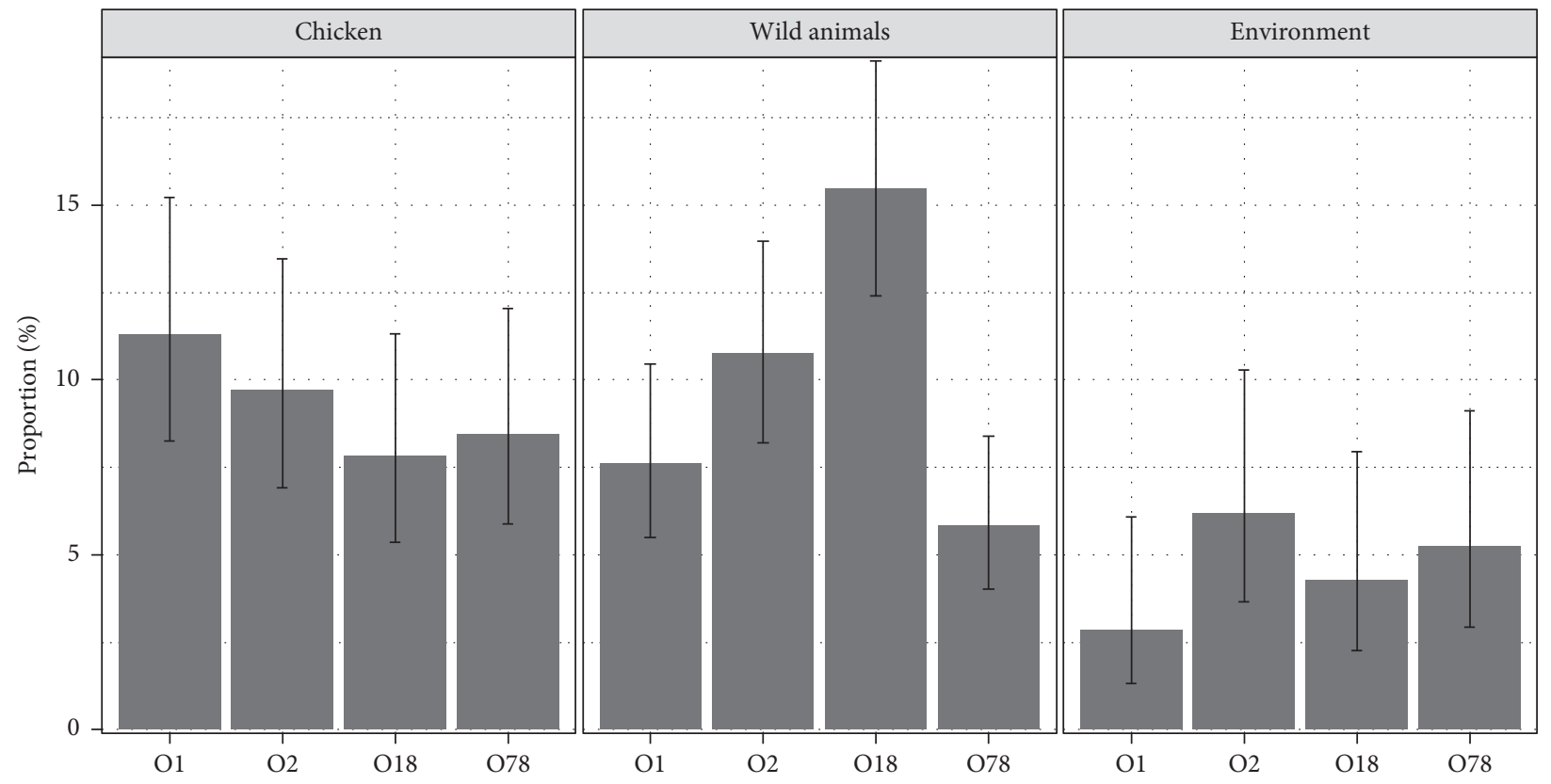

(b)

Figure 2: A pie chart showing the distribution of serotypes O1, O2, O18, and O78 per all E. coli isolates (a) and bar graphs showing proportions of serotypes $\mathrm{O} 1, \mathrm{O} 2, \mathrm{O} 18$, and $\mathrm{O} 78$ per chicken, wild animals, and environment (b). The error bar indicates $95 \%$ confidence interval.

and $39 \mathrm{O} 78$ isolates showed that $E$. coli present in the settings of poultry husbandry were highly divergent. Multiple subclusters were assigned within each serotype population $(\mathrm{O} 1$ : 38 clusters, $\mathrm{O} 2: 35$ clusters, $\mathrm{O} 18: 64$ clusters, and $\mathrm{O} 78: 35$ clusters). The number of subclusters per serotype in this study is obviously higher than that observed in previous investigations using the same method [40-42]. More heterogeneity of clustering patterns in our study might result from a larger number of isolates or variety of sample sources included in our analysis or might be due to the more rapid evolution of E. coli in the Mekong Delta [31, 32]. As a result, this study reaffirms that $E$. coli existing in the poultry husbandry in Vietnam has undergone large and rapid genetic diversification.
This study places greater emphasis on inferring genetic relationship and cross-contamination potentials of $E$. coli between different host species and environment on the basis of DNA fingerprints generated from ERIC-PCR. It is evident from the four O1, O2, O18, and O78 fingerprint dendrograms that several $E$. coli strains collected from chickens, wild animals, and environment were grouped in the same subclusters with high genetic similarities (Figures 3(a)$3(\mathrm{~d}))$. This result suggests that there might be cross transmission of $E$. coli between different sources in the poultry husbandry setting. Previously, several studies also demonstrated that fecal contamination from poultry is the primary dissemination and transmission source of $E$. coli to environment and surrounding animals $[43,44]$. In fact, from our 


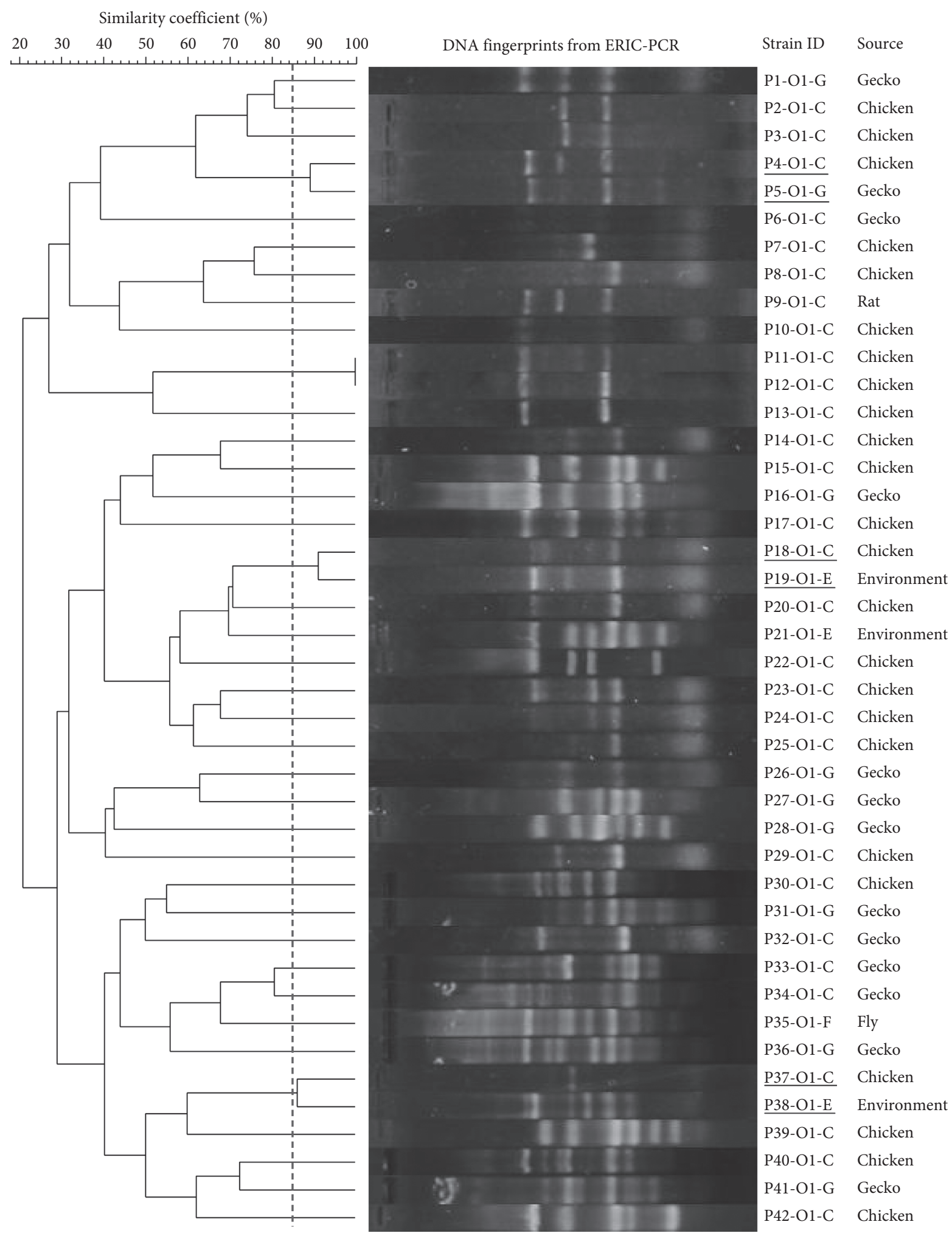

(a)

Figure 3: Continued. 


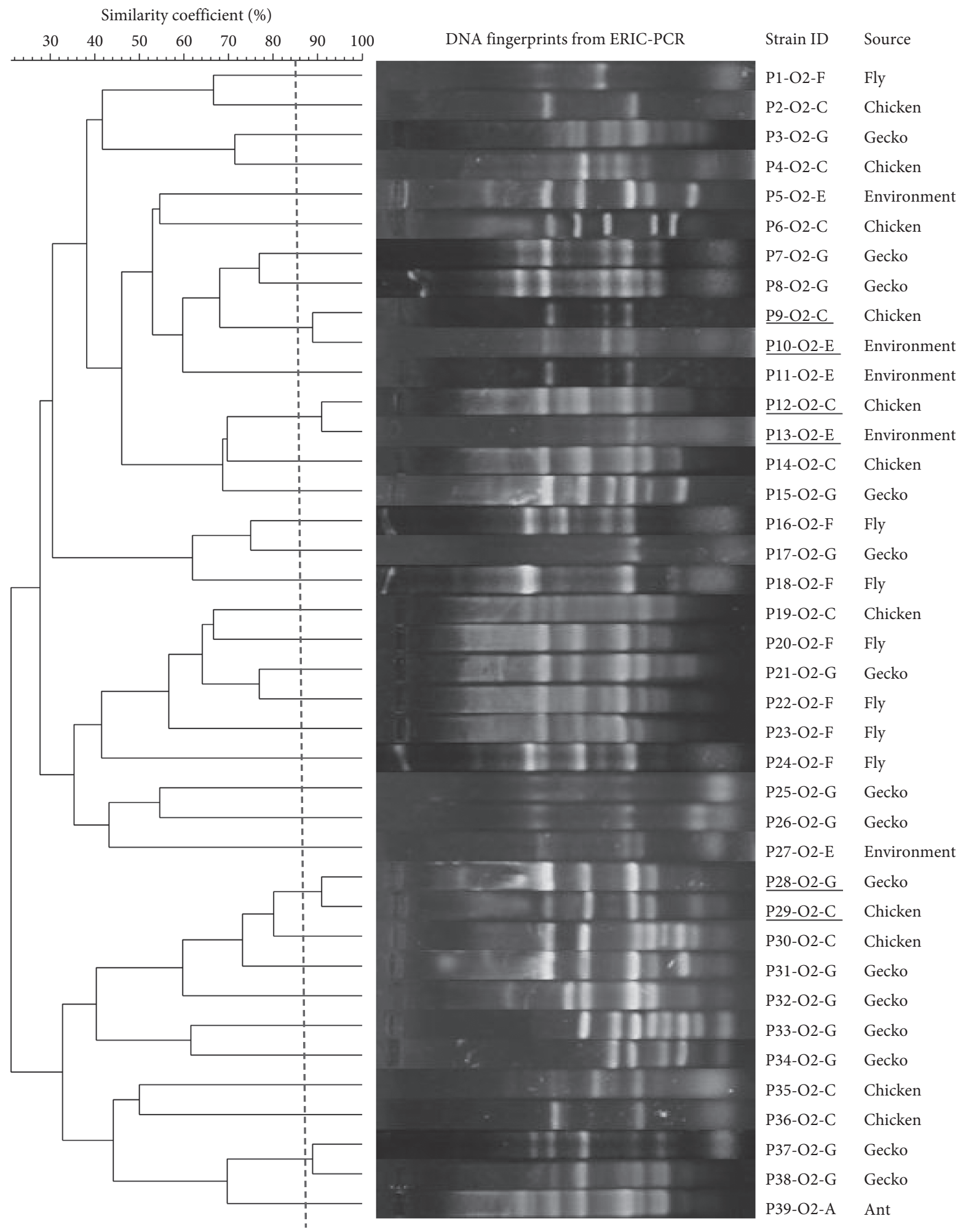

(b)

Figure 3: Continued. 


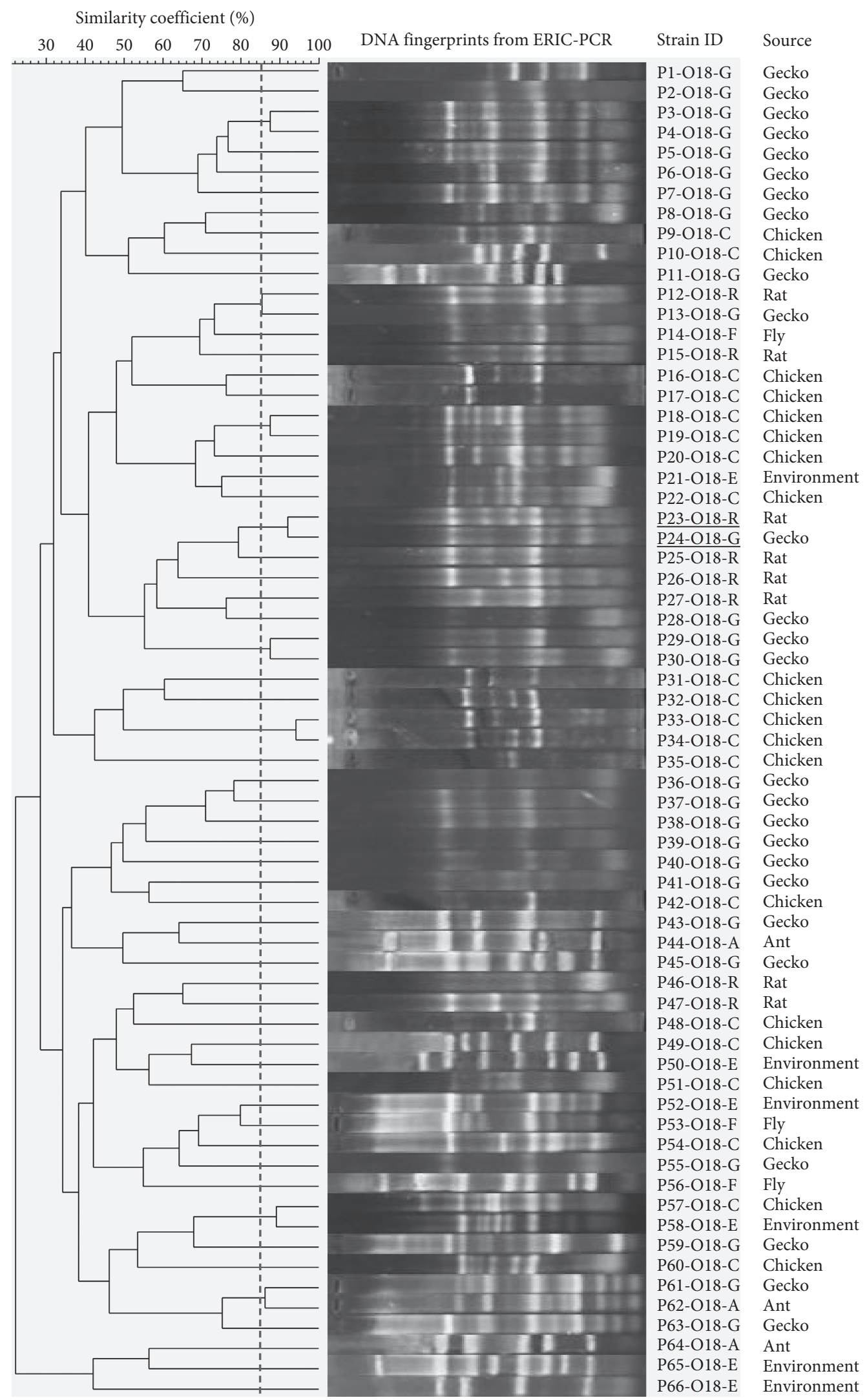

(c)

FIgURE 3: Continued. 


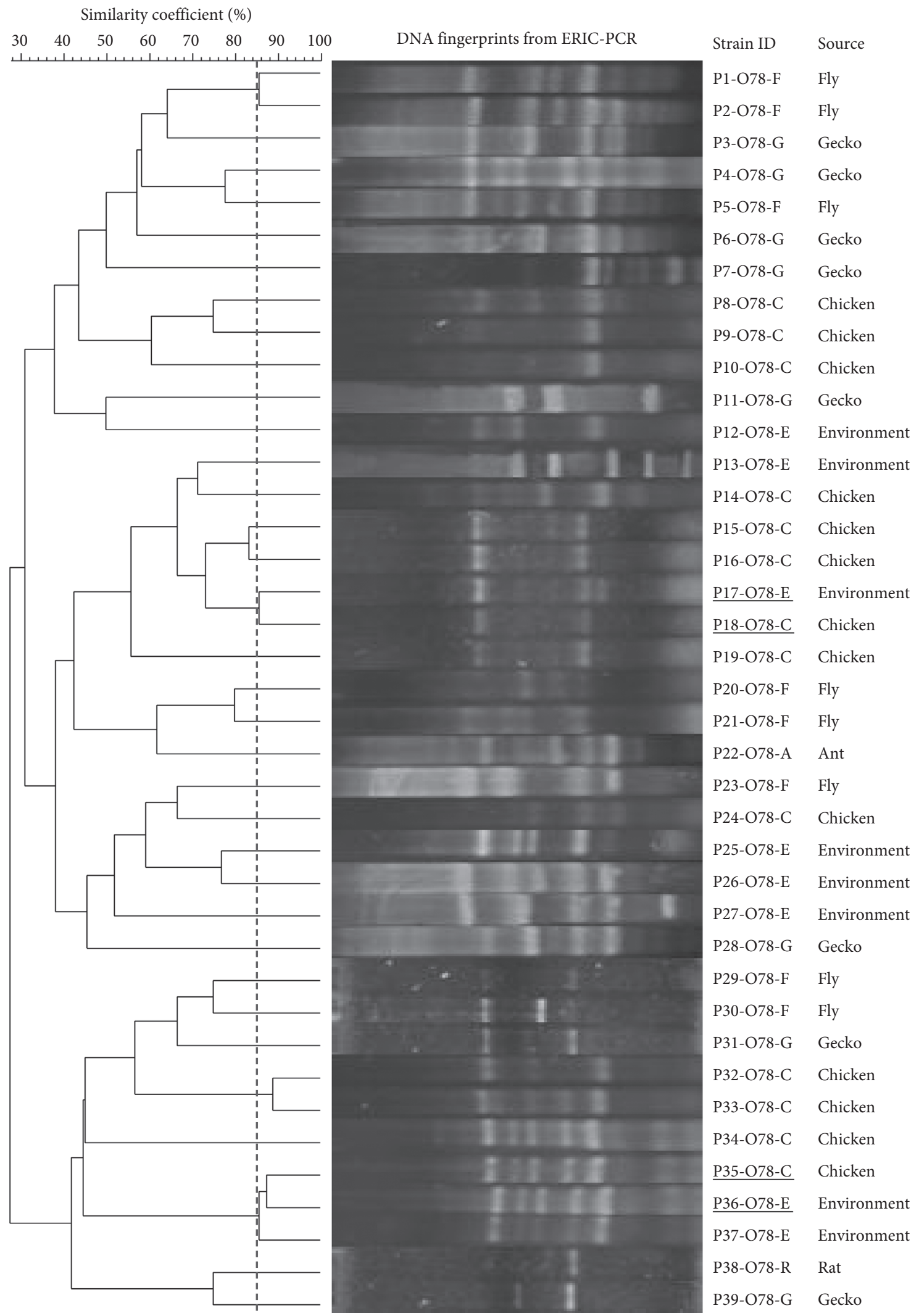

(d)

Figure 3: Dendrograms and genomic DNA fingerprints of E. coli isolates that belong to the O1 (a), O2 (b), O18 (c), and O78 (d). The results were produced from ERIC-PCR and Bionumerics software version 7.0. The dashed vertical line indicates the cutoff value $\geq 85 \%$ genetic similarity coefficient. E. coli strains that were isolated from different sample sources and shared $\geq 85 \%$ genetic similarity coefficient were underlined. 
empirical observation, almost all small/backyard farms in the Mekong Delta have relatively poor hygiene practices, and such wild animals as geckos, rats, flies, and ants commonly exist in poultry farms. Thus, cross transmission is almost certain. This study suggests that monitoring E. coli as well as APEC contamination in environment and in-farms wild animals could be considered as an indicator of interspecies barrier transposition in poultry production. In addition, the observed high prevalence of $E$. coli belonging to the $\mathrm{O} 1, \mathrm{O} 2$, $\mathrm{O} 18$, and $\mathrm{O} 78$ serotypes from wild animals in poultry farms is also concerning since these species also play important roles in food-borne disease transmission but they are usually neglected in routine surveillance programs $[36,38]$.

This study successfully examined in part the population structure of E. coli with the four common APEC serotypes and provided genetic evidence for cross transmission of these E. coli serotypes between different sources in the poultry husbandry. However, there are some inevitable limitations in the present study; for example, virulence, pathotypes, and antibiotics resistance characteristics of the obtained $E$. coli were not examined or a large proportion of E. coli was not serotyped. Nevertheless, this study added significant information on biological characteristics and epidemiological dynamics of E. coli which is necessary for better control of avian colibacillosis and prevention of the threat of zoonotic diseases caused by APEC.

\section{Conclusion}

E. coli was predominantly isolated from small/backyard poultry farms. Serotypes O1, O2, O18, and O78 were accounted for a high proportion of the E. coli population and O18 was the predominant serotype among the examined E. coli. Chickens, in-farms geckos, and rats were identified as the major sources of dissemination and transmission of E. coli. Polymorphic DNA fingerprint analyses revealed that APEC from different sources of poultry husbandry exhibit large genetic heterogeneity and closed genetic relationship. Therefore, this study provides important information about epidemiological and genetic characteristics of E. coli and emphasizes the necessity of stricter biocontainment in poultry production for prevention and control of avian colibacillosis caused by APEC.

\section{Data Availability}

All data generated or analyzed during this study are included in this published article and its supplementary information files.

\section{Conflicts of Interest}

The authors declare no conflicts of interest.

\section{Authors' Contributions}

Lam Thanh Nguyen and Nguyen Khanh Thuan contributed equally to this work.

\section{Acknowledgments}

This study was funded in part by the Can Tho University Improvement Project VN14-P6 and supported by the Japanese ODA Loan.

\section{Supplementary Materials}

Supplementary Table 1. Summary of sample types and their distribution. Supplementary Figure 1. Geographical map showing the location of sampling site: Vinh Long Province (black) is situated in the center of the Mekong Delta (grey) on the Vietnam map. Supplementary Figure 2. The PCR products amplified using the allele-specific genes of O1 (3A), O2 (3-B), O18 (3-C), and O78 (3-D) serotypes. Lane M: 100 bp DNA ladder; lane P: positive control; lane N: negative control (distilled water as template); lane 1 to lane 6: DNA samples of $E$. coli tested. Arrows indicate sizes of amplified DNA fragments as follows: $263 \mathrm{bp}(\mathrm{O} 1), 355 \mathrm{bp}$ (O2), $459 \mathrm{bp}$ (O18), and 623 bp (O78). . (Supplementary Materials)

\section{References}

[1] O. Tenaillon, D. Skurnik, B. Picard, and E. Denamur, "The population genetics of commensal Escherichia coli," Nature Reviews Microbiology, vol. 8, no. 3, pp. 207-217, 2010.

[2] J. B. Kaper, J. P. Nataro, and H. L. T. Mobley, "Pathogenic Escherichia coli," Nature Reviews Microbiology, vol. 2, no. 2, pp. 123-140, 2004.

[3] L. K. Nolan, J. P. Vaillancourt, N. L. Barbieri, and C. M. Logue, "Colibacillosis," Diseases of Poultry, 14th Edition, Chapter 18, pp. 770-830, John Wiley \& Sons, Inc., Hoboken, NJ, USA, 2020.

[4] K. E. Rodriguez-Siek, C. W. Giddings, C. Doetkott, T. J. Johnson, M. K. Fakhr, and L. K. Nolan, "Comparison of Escherichia coli isolates implicated in human urinary tract infection and avian colibacillosis," Microbiology (Reading), vol. 151, no. 6, pp. 2097-2110, 2005.

[5] M. Moulin-Schouleur, M. Répérant, S. Laurent et al., "Extraintestinal pathogenic Escherichia coli strains of avian and human origin: link between phylogenetic relationships and common virulence patterns," Journal of Clinical Microbiology, vol. 45, no. 10, pp. 3366-3376, 2007.

[6] M. Mellata, "Human and avian extraintestinal PathogenicEscherichia coli: infections, zoonotic risks, and antibiotic resistance trends," Foodborne Pathogens and Disease, vol. 10, no. 11, pp. 916-932, 2013.

[7] D. Kathayat, D. Lokesh, S. Ranjit, and G. Rajashekara, “Avian pathogenic Escherichia coli (APEC): an overview of virulence and pathogenesis factors, zoonotic potential, and control strategies," Pathogens, vol. 10, no. 4, p. 467, 2021.

[8] F. Kauffmann, "The serology of the coli group," The Journal of Immunology, vol. 57, pp. 71-100, 1947.

[9] B. Liu, A. Furevi, A. V. Perepelov et al., "Structure and genetics of Escherichia coli O antigens," FEMS Microbiology Reviews, vol. 44, no. 6, pp. 655-683, 2019.

[10] F. Scheutz, T. Cheasty, D. Woodward, and H. R. Smith, "Designation of O174 and O175 to temporary O groups OX3 and OX7, and six new E. coli $\mathrm{O}$ groups that include Verocytotoxin-producing E. coli (VTEC): O176, O177, O178, O179, O180 and O181," Apmis, vol. 112, no. 9, pp. 569-584, 2004. 
[11] C. Ewers, G. Li, H. Wilking et al., "Avian pathogenic, uropathogenic, and newborn meningitis-causing Escherichia coli: how closely related are they?" International Journal of Medical Microbiology, vol. 297, no. 3, pp. 163-176, 2007.

[12] S. Huja, Y. Oren, E. Trost et al., "Genomic avenue to avian colisepticemia," MBio, vol. 6, 2015.

[13] J. W. Mehat, A. H. M. van Vliet, and R. M. La Ragione, "The avian pathogenic Escherichia coli (APEC) pathotype is comprised of multiple distinct, independent genotypes," Avian Pathology, vol. 50, no. 5, pp. 402-416, 2021.

[14] J. E. Blanco, M. Blanco, A. Mora et al., "Serotypes of Escherichia coli isolated from septicaemic chickens in Galicia (northwest Spain)," Veterinary Microbiology, vol. 61, no. 3, pp. 229-235, 1998.

[15] X. M. Wang, X.-P. Liao, W.-J. Zhang et al., "Prevalence of serogroups, virulence genotypes, antimicrobial resistance, and phylogenetic background of avian PathogenicEscherichia coliin South of China," Foodborne Pathogens and Disease, vol. 7, no. 9, pp. 1099-1106, 2010.

[16] Y. B. Kim, M. Y. Yoon, J. S. Ha et al., "Molecular characterization of avian pathogenic Escherichia coli from broiler chickens with colibacillosis," Poultry Science, vol. 99, no. 2, pp. 1088-1095, 2020.

[17] J.-D. Cesaro, H. Nguyen, and D. Guillaume, Atlas of livestock transitions in Vietnam (1986-2016), IPSARD-CIRAD, Hanoi, 65p. ISBN 978-2-87614-746-1, 2020.

[18] GSO, Statistical Yearbook of Vietnam. 2020, General Statistical Office of Vietnam, Hanoi, Vietnam, 2020.

[19] Vietnam National Standard (TCVN), Microbiology of Food and Animal Feeding Stuffs-Horizontal Method for the Enumeration of $\beta$-glucuronidase-positive Escherichia coli-Part 2: Colony-count Technique at $44^{\circ} \mathrm{C}$ Using 5-Bromo-4-Chloro-3Indolyl $\beta$-D-glucuronide, Vietnam National Standard, Vietnam, 2008.

[20] S. T. Cowan, Cowan and Steel's Manual for the Identification of Medical Bacteria, Cambridge University Press, Cambridge, UK, 2004.

[21] T. L. K. Ly, T. P. Tran, T. T. Nguyen, and T. Iwata, "Prevalence of Escherichia coli $\mathrm{O} 157$ from cattle and foods in the Mekong delta, Vietnam," Journal of Veterinary Medical Science, vol. 13, pp. 107-113, 2009.

[22] T. T. Hong To, H. Yanagawa, N. Khanh Thuan et al., "Prevalence of Vibrio parahaemolyticus causing acute hepatopancreatic necrosis disease of shrimp in shrimp, Molluscan shellfish and water samples in the Mekong Delta, Vietnam," Biology (Basel), vol. 9, p. 312, 2020.

[23] S. Wang, Q. Meng, J. Dai et al., "Development of an allelespecific PCR assay for simultaneous sero-typing of avian pathogenic Escherichia coli predominant O1, O2, O18 and O78 strains," PLoS One, vol. 9, Article ID e96904, 2014.

[24] J. Versalovic, T. Koeuth, and J. R. Lupski, "Distribution of repetitive DNA sequences in eubacteria and application to fingerprinting of bacterial genomes," Nucleic Acids Research, vol. 19, pp. 6823-6831, 1991.

[25] E. A. Casarez, S. D. Pillai, and G. D. Di Giovanni, "Genotype diversity of Escherichia coli isolates in natural waters determined by PFGE and ERIC-PCR," Water Research, vol. 41, pp. 3643-3648, 2007.

[26] R Core Team, R: A Language and Environment for Statistical Computing, R Foundation for Statistical Computing, Vienna, Austria, 2013.

[27] H. Wickham, Ggplot2: Elegant Graphics for Data Analysis, Springer, Berlin, Germany, 2016.
[28] S. Dorai-Raj, "Binom: Binomial Confidence Intervals for Several Parameterizations," $R$ package version, vol. 1, pp. 1-1, 2014.

[29] M. Stevenson, M. M. Stevenson, and I. BiasedUrn, Package 'epiR', 2021.

[30] R. Guabiraba and C. Schouler, "Avian colibacillosis: still many black holes,” FEMS Microbiology Letters, vol. 362, no. 15, 2015.

[31] V. T. Nguyen, J. J. Carrique-Mas, T. H. Ngo et al., "Prevalence and risk factors for carriage of antimicrobial-resistant Escherichia coli on household and small-scale chicken farms in the Mekong Delta of Vietnam," Journal of Antimicrobial Chemotherapy, vol. 70, pp. 2144-2152, 2015.

[32] N. Nhung, N. Cuong, J. Campbell et al., "High levels of antimicrobial resistance among Escherichia coli isolates from livestock farms and synanthropic rats and shrews in the Mekong Delta of Vietnam," Antimicrobial Agents and Chemotherapy, vol. 81, pp. 812-820, 2015.

[33] M. Dho-Moulin and J. M. Fairbrother, "Avian pathogenic Escherichia coli (APEC)," Veterinary Research, vol. 30, pp. 299-316, 1999.

[34] S. M. Lutful Kabir, "Avian colibacillosis and salmonellosis: a closer look at epidemiology, pathogenesis, diagnosis, control and public health concerns," International Journal of Environmental Research and Public Health, vol. 7, pp. 89-114, 2010.

[35] M. Moulin-Schouleur, C. Schouler, P. Tailliez et al., "Common virulence factors and genetic relationships between O18: K1: H7 Escherichia coli isolates of human and avian origin," Journal of Clinical Microbiology, vol. 44, pp. 3484-3492, 2006.

[36] B. J. Morrison and J. E. Rubin, "Detection of multidrug-resistant Gram-negative bacteria from imported reptile and amphibian meats," Journal of Applied Microbiology, vol. 129, pp. 1053-1061, 2020.

[37] C. Marin, L. Lorenzo-Rebenaque, O. Laso, J. Villora-Gonzalez, and S. Vega, "Pet reptiles: a potential source of transmission of multidrug-resistant Salmonella," Frontiers in Veterinary Science, vol. 7, 2021.

[38] K. T. Nguyen, M. Hasegawa, T. M. T. Vo et al., "Wild geckos considered as the natural reservoir of Salmonella Weltevreden in Southeast Asian countries," Zoonoses and Public Health, vol. 68, no. 7, pp. 815-822, 2021.

[39] K. J. Meacham, L. Zhang, B. Foxman, R. J. Bauer, and C. F. Marrs, "Evaluation of genotyping large numbers of Escherichia coli isolates by enterobacterial repetitive intergenic consensus-PCR," Journal of Clinical Microbiology, vol. 41, pp. 5224-5226, 2003.

[40] B. R. Mohapatra, K. Broersma, and A. Mazumder, "Comparison of five rep-PCR genomic fingerprinting methods for differentiation of fecal Escherichia coli from humans, poultry and wild birds," FEMS Microbiology Letters, vol. 277, pp. 98-106, 2007.

[41] A. Hussain, S. Shaik, A. Ranjan et al., "Risk of transmission of antimicrobial resistant Escherichia coli from commercial broiler and free-range retail chicken in India," Frontiers in Microbiology, vol. 8, 2017.

[42] M. S. Sekhar, N. M. Sharif, T. S. Rao, and M. Metta, "Genotyping of virulent Escherichia coli obtained from poultry and poultry farm workers using enterobacterial repetitive intergenic consensus-polymerase chain reaction," Veterinary World, vol. 10, pp. 1292-1296, 2017.

[43] H. Duan, T. Chai, Y. Cai, Z. Zhong, M. Yao, and X. Zhang, "Transmission identification of Escherichia coli aerosol in chicken houses to their environments using ERIC-PCR," 
Science in China-Series C: Life Sciences, vol. 51, pp. 164-173, 2008.

[44] H. Laube, A. Friese, C. von Salviati, B. Guerra, and U. Rösler, "Transmission of ESBL/AmpC-producing Escherichia coli from broiler chicken farms to surrounding areas," Veterinary Microbiology, vol. 172, pp. 519-527, 2014. 